\title{
Gestational diabetes induces alterations of sirtuins in fetal endothelial cells
}

\author{
Juan Gui ${ }^{1}, 2$, Arne Potthast ${ }^{3}$, Anne Rohrbach'1, Katja Borns ${ }^{1}$, Anibh M. Das ${ }^{3}$ and Frauke von Versen-Höynck ${ }^{1}$
}

BACKGROUND: Gestational diabetes (GDM) has long-term consequences for the offspring. Sirtuins (SIRTs) are associated with vascular and metabolic functions. We studied the impact of GDM on SIRT activity and expression in fetal endothelial colony-forming cells (ECFCs) and human umbilical vein endothelial cells (HUVECs) from pregnancies complicated by GDM. METHODS: ECFCS and HUVECS were isolated from cord and cord blood of 10 uncomplicated pregnancies (NPs) and 10 GDM pregnancies. Nicotinamidadenindinukleotid (NAD+) concentration, SIRT1 and SIRT3 activity, transcription levels of SIRT1, SIRT3, and SIRT4, and protein levels of SIRT1, SIRT3, and SIRT4 were determined in vitro with or without SIRT activators resveratrol (RSV) and paeonol.

RESULTS: Fetal ECFCS from GDM pregnancies showed a decreased NAD ${ }^{+}$concentration, reduced SIRT1 and SIRT3 activity, and lower transcription levels of SIRT1, SIRT3, and SIRT4. HUVECs from GDM pregnancies had decreased NAD+ concentrations and transcription levels of SIRT1 and SIRT4. RSV markedly enhanced the expression and activity of SIRTs in ECFCS and HUVECs, while paeonol was active only in ECFCs.

CONCLUSION: A reduction of SIRT activity and expression in fetal endothelial cells provides potential mechanistic insights into the pathophysiology of long-term cardiovascular complications observed in the offspring of GDM pregnancies. SIRT activators can increase SIRT activity in ECFCs, which opens perspectives for new therapeutic targets.

A dverse events during fetal life have been implicated to induce fetal programming, which can result in chronic diseases (1). Especially gestational diabetes (GDM) of the mother has been associated with cardiovascular disease, metabolic syndrome, and obesity during later life in the offspring $(2,3)$. The pathophysiological processes responsible for these late complications are largely unknown.

Sirtuins (SIRTs) are important regulators of aging and metabolic diseases $(4,5)$. Mammalian SIRTs belong to the histone deacetylase class III family comprising seven members (SIRT1$7)$, which require Nicotinamidadenindinukleotid $\left(\mathrm{NAD}^{+}\right)$for their enzymatic activity (6). SIRT1 downregulation has been observed in peripheral blood mononuclear cells of individuals with impaired glucose tolerance $(7,8)$. SIRT1 is known to regulate vascular endothelial cell functions (9). Furthermore, in vitro and in vivo studies demonstrated that a reduction in the number of endothelial progenitor cells (EPCs) under hyperglycemia is associated with reduced SIRT1 levels and activity $(8,10)$. Endothelial cells regulate vascular tone and are the first fetal cells exposed to maternal hyperglycemia.

In recent years, it has become evident that SIRT1 is a key player of EPC dysfunction in insulin resistance and metabolic syndrome $(7,10,11)$. Overexpression of endothelium-specific SIRT1 protects against endothelial dysfunction induced by a high-fat diet and hyperglycemia $(12,13)$. Recently, resveratrol (RSV) and paeonol (2'-Hydroxy-4'-methoxyacetophenone) have been shown to activate SIRTs and thus can be used for pharmacological interventions $(14,15)$. Activation of SIRT1 by RSV can improve vascular functions (16), while paeonol prevented premature senescence induced by oxidative stress in human umbilical vein endothelial cells (HUVECs) by modulating the expression of SIRT1 (15).

EPCs derived from bone marrow contribute to endothelial repair (17). Among them, the specific subtype of endothelial colony-forming cells (ECFCs) harbor clonogenic potential, endothelial phenotype, have the capacity to incorporate into neovessels, and participate in the maintenance of vascular homeostasis in vivo. Impairment in the number and function of fetal ECFCs has been observed in preeclampsia and $\operatorname{GDM}(18,19)$.

The purpose of this study was to investigate whether fetal endothelial cells including ECFCs and HUVECs from GDM pregnancies show alterations of SIRTs compared with uncomplicated pregnancies (NPs). We further investigated whether paeonol or RSV can activate SIRTs in ECFCs and HUVECs from GDM pregnancies.

\section{RESULTS}

\section{Patient Demographics}

There were no differences in maternal age, pre-pregnancy body mass index (BMI), gestational age at delivery, cesarean section rate, gender, birth weight, arterial cord blood $\mathrm{pH}$, and

\footnotetext{
The first two authors and the last two authors contributed equally to this work.

'Department of Obstetrics and Gynecology, Hannover Medical School, Hannover, Germany; ${ }^{2}$ Department of Obstetrics and Gynecology, Tongji Hospital, Tongji Medical College, Huazhong University of Science and Technology, Wuhan, Hubei, China; ${ }^{3}$ Department of Pediatrics, Hannover Medical School, Hannover, Germany. Correspondence:

Frauke von Versen-Höynck (vonversen-hoeynck.frauke@mh-hannover.de)

Received 21 February 2015; accepted 16 October 2015; advance online publication 17 February 2016. doi:10.1038/pr.2015.269
} 
glycated hemoglobin (HbA1c) levels between women with NPs and pregnancies complicated by GDM (Table 1).

\section{Reduction of $\mathrm{NAD}^{+}$, SIRT Activity, and Expression in ECFCs From GDM Pregnancies}

$\mathrm{NAD}^{+}$concentration in ECFCs from GDM pregnancies was significantly lower $(0.72 \pm 0.24, P=0.02)$ compared with NPs

Table 1. Clinical and demographic data of patients

\begin{tabular}{lccc}
\hline & NPs & GDM & $P$ \\
\cline { 2 - 3 } Variables & $(n=10)$ & $(n=10)$ & Value \\
\hline Maternal age (y) & $30.20 \pm 5.49$ & $34.10 \pm 4.63$ & 0.10 \\
Primiparous (\%) & $40 \%$ & $60 \%$ & 0.6 \\
Gestational age at delivery (wk) & $39.63 \pm 1.18$ & $39.19 \pm 1.34$ & 0.44 \\
Maternal pre-pregnancy BMI $\left(\mathrm{kg} / \mathrm{m}^{2}\right)$ & $27.86 \pm 6.82$ & $30.70 \pm 7.34$ & 0.38 \\
Caesarean delivery (\%) & $50 \%$ & $40 \%$ & 1.00 \\
Birth weight (g) & $3615 \pm 445.1$ & $3679 \pm 517.5$ & 0.77 \\
Baby gender, male $n(\%)$ & $6(60 \%)$ & $2(20 \%)$ & 0.17 \\
Arterial cord pH & $7.27 \pm 0.09$ & $7.26 \pm 0.05$ & 0.88 \\
HbA1c before delivery & $5.09 \pm 0.36$ & $5.27 \pm 0.26$ & 0.24 \\
\hline
\end{tabular}

Data are given as mean \pm SD or number (percentage).

$\mathrm{BMI}$, body mass index; GDM, gestational diabetes; $\mathrm{HbA} 1 \mathrm{c}$, glycated hemoglobin;

NPs, uncomplicated pregnancies. (control $=1)$, as well as SIRT1 activity $(0.87 \pm 0.28, P=0.004)$, SIRT3 activity $(0.78 \pm 0.48, P=0.03)$, and relative transcription levels of SIRT1 $(0.65 \pm 0.29, P=0.03)$, SIRT3 (0.81 \pm 0.16 , $P=0.003)$, and SIRT4 $(0.75 \pm 0.23, P=0.003)$ when compared with NPs (Figure 1). There were no differences in protein levels of SIRT1 $(P=0.44)$, SIRT3 $(P=0.38)$, and SIRT4 $(P=0.84)$ between the two groups.

\section{Reduction of NAD ${ }^{+}$, SIRT Activity, and Expression in HUVECs From GDM Pregnancies}

HUVECs from GDM pregnancies had a significantly lower $\mathrm{NAD}^{+}$concentration $(0.85 \pm 0.21, P=0.006)$ and relative transcription levels of SIRT1 $(0.85 \pm 0.14, P=0.04)$ and SIRT4 $(0.69 \pm 0.11, P=0.0039)$ when compared with NPs (control $=1)$. There were no differences in SIRT1 activity $(1.11 \pm 0.29$, $P=0.34)$, SIRT3 activity $(1.21 \pm 0.38, P=0.30)$, and relative transcription levels of SIRT3 $(0.94 \pm 0.35, P=0.70)$ (Figure 2). The protein levels of SIRT1 $(P=0.08)$, SIRT3 $(P=0.15)$, and SIRT4 $(P=0.97)$ were also not different between the two groups.

\section{Hyperglycemia In Vitro Resulted in Elevated NAD+ Levels and a} Reduction of SIRT Activity and Expression in ECFCs

In ECFCs, the $\mathrm{NAD}^{+}$concentration was significantly increased after treatment with $30-\mathrm{mM}$ glucose (15 mM: $1.28 \pm 0.22$,

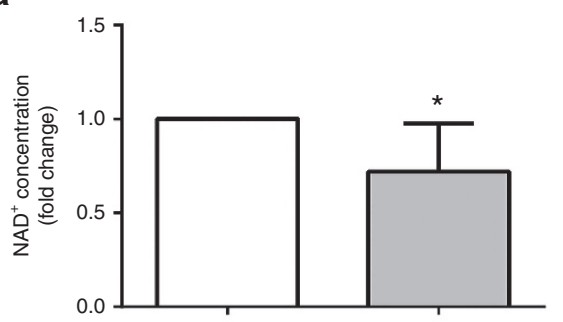

d

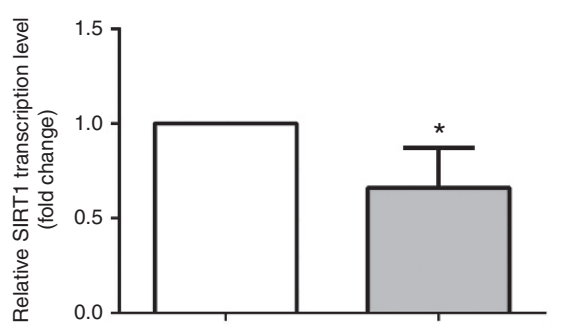

g

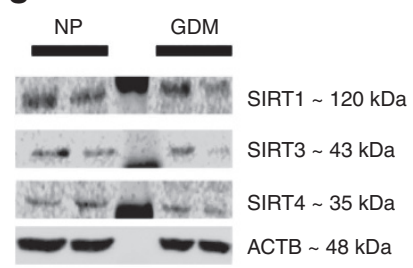

b

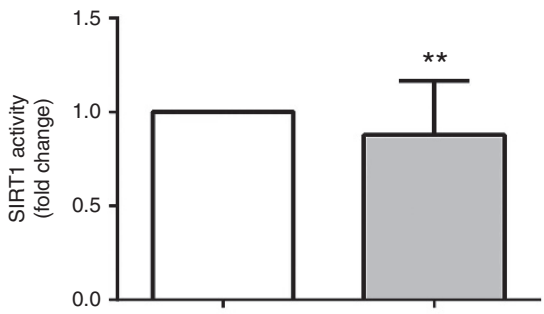

e

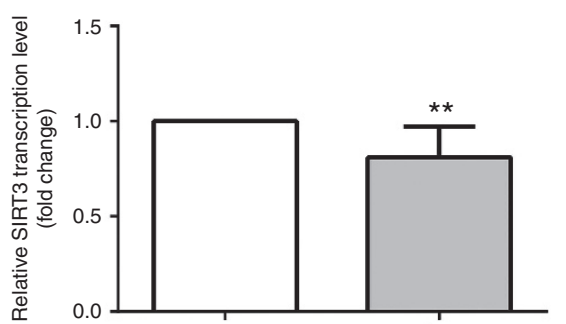

i
C

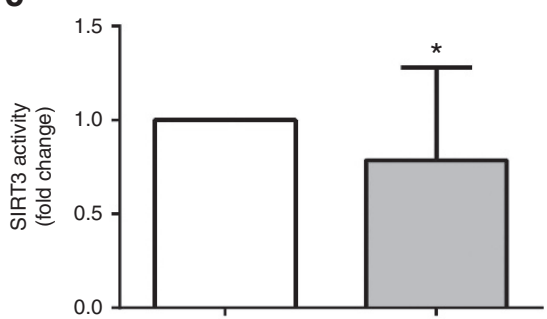

f

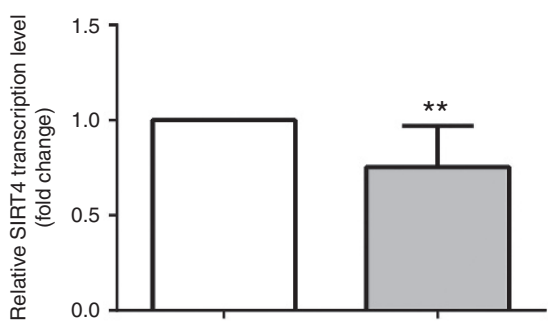

j

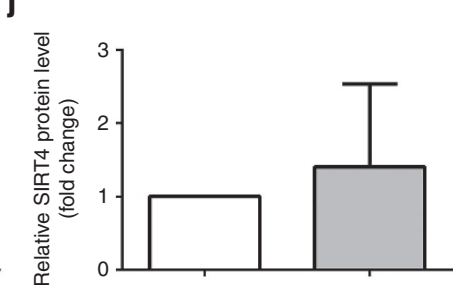

Figure 1. Alterations of sirtuins (SIRTs) in endothelial colony-forming cells (ECFCs) from gestational diabetes (GDM) pregnancies (gray) compared with uncomplicated pregnancies (NPs; white). (a) NAD+ concentration, (b) SIRT1 activity, (c) SIRT3 activity, relative transcription levels of (d) SIRT1, (e) SIRT3, and (f) SIRT4, and (g) representative immunoblots of ECFCs from NPs and GDM pregnancies. Relative protein levels of (h) SIRT1, (i) SIRT3, and (j) SIRT4 of ECFCs from NPs compared with GDM pregnancies were not significantly different. $(n=10)$. Data are presented as mean fold changes \pm SD of GDM pregnancies as compared with NPs (control $=1) .{ }^{*} P<0.05$ or ${ }^{* *} P<0.01$ when compared with NPs. 


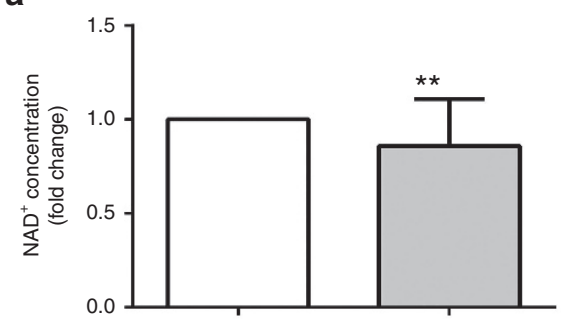

d

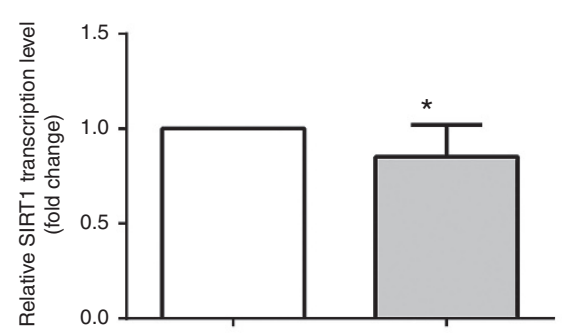

g

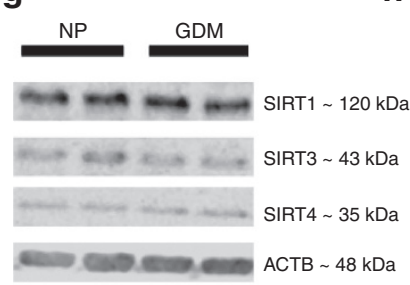

b

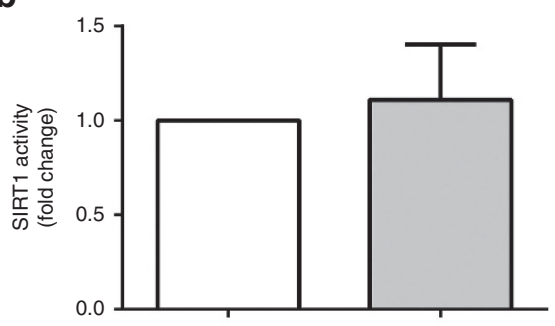

e

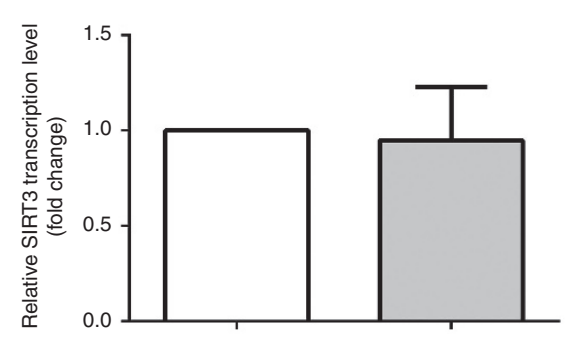

i c

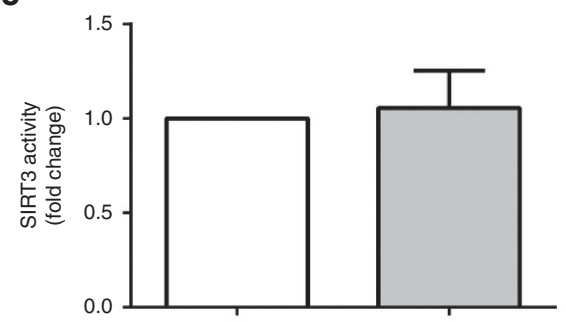

f

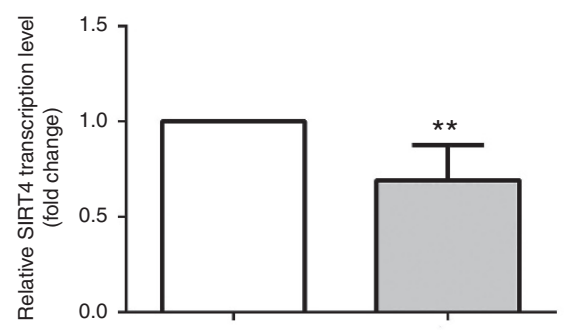

j
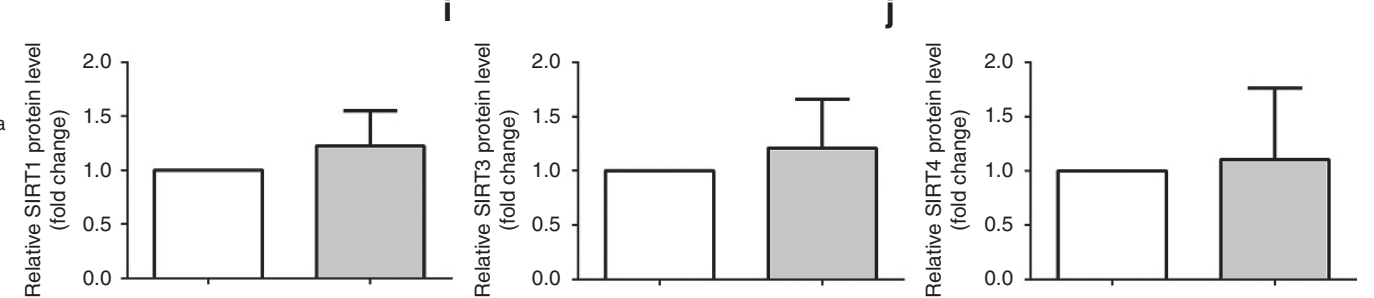

Figure 2. Alterations of sirtuins (SIRTs) in human umbilical vein endothelial cells (HUVECs) from gestational diabetes (GDM) pregnancies (gray) compared with uncomplicated pregnancies (NPs; white). (a) NAD concentration, (b) SIRT1 activity and (c) SIRT3 activity, relative transcription levels of (d) SIRT1, (e) SIRT3, and (f) SIRT4, and (g) representative immunoblots of HUVECs from NPs and GDM pregnancies. Relative protein levels of (h) SIRT1, (i) SIRT3, and (j) SIRT4 of HUVECs from NPs compared with GDM pregnancies without significant alterations. $(n=6)$. Data are presented as mean fold changes \pm SD of GDM pregnancies as compared with NPs (control $=1),{ }^{*} P<0.05$ or ${ }^{* *} P<0.01$ when compared with NPs.

$P>0.05 ; 1.64 \pm 0.23, P<0.05)$ compared with standard culture medium containing $5.5-\mathrm{mM}$ glucose $($ control $=1)$. Incubation with $30-\mathrm{mM}$ glucose decreased SIRT1 activity (15 mM: $1.13 \pm 0.25, P>0.05$; $30 \mathrm{mM}$ : $0.55 \pm 0.11, P<0.05)$. We observed no change in SIRT3 activity ( $15 \mathrm{mM}: 1.13 \pm 0.18$, $P>0.05 ; 30 \mathrm{mM}$ : $0.65 \pm 0.26, P>0.05$ ) (Figure 3). SIRT1 (15 mM: $1.10 \pm 0.11, P>0.05 ; 30 \mathrm{mM}: 0.77 \pm 0.13, P<0.05)$ and SIRT4 transcription levels ( $15 \mathrm{mM}: 1.26 \pm 0.19, P>0.05 ; 30 \mathrm{mM}$ : $0.69 \pm 0.08, P<0.05)$ were significantly lower after exposure to $30-\mathrm{mM}$ glucose, while there was no change in SIRT3 transcription levels (15 mM: $1.15 \pm 0.12, P>0.05 ; 30 \mathrm{mM}$ : $0.83 \pm 0.09$, $P>0.05)$. There were no differences in protein levels of SIRT1, SIRT3, and SIRT4 between control cells, 15-mM treatment, and $30-\mathrm{mM}$ treatment.

\section{Hyperglycemic Treatment In Vitro Resulted in a Reduction of SIRT Activity and Expression in HUVECs}

$\mathrm{NAD}^{+}$concentration in HUVECs remained unaffected after a treatment with $15-\mathrm{mM}$ or $30-\mathrm{mM}$ glucose (15 mM: $1.0 \pm 0.10$, $P>0.05 ; 30$ mM: $1.13 \pm 0.18, P>0.05)$ compared with standard culture medium containing $5.5-\mathrm{mM}$ glucose $($ control $=1$ ). As opposed to this, exposure to $30-\mathrm{mM}$ glucose resulted in a significant decrease of SIRT1 $(15 \mathrm{mM}$ : $0.90 \pm 0.03 P>0.05$;
$30 \mathrm{mM}: 0.73 \pm 0.08, P<0.01)$ and SIRT3 activity $(15 \mathrm{mM}$ : $0.90 \pm 0.02, P>0.05 ; 30 \mathrm{mM}: 0.59 \pm 0.13, P<0.01$ ) (Figure 4). SIRT1 (15 mM: $1.04 \pm 0.41, P>0.05 ; 30 \mathrm{mM}: 0.77 \pm 0.21$, $P<0.05)$ and SIRT4 $(15 \mathrm{mM}: 0.86 \pm 0.16, P>0.05 ; 30 \mathrm{mM}$ : $0.78 \pm 0.09, P<0.05)$ transcription levels showed a significant reduction after a 30 -mM glucose treatment, whereas there were no alterations in SIRT3 (15 mM: $1.26 \pm 0.12, P>0.05 ; 30 \mathrm{mM}$ : $0.89 \pm 0.18, P>0.05)$ transcription levels. SIRT1, SIRT3, and SIRT4 protein levels showed no differences between control cells, $15-\mathrm{mM}$ glucose treatments, and $30-\mathrm{mM}$ glucose treatments.

\section{Effect of SIRT Activators in ECFCs}

In this section, the control group represents cells mock treated with ethanol from each patient group. After treatment with $50-\mu \mathrm{M}$ paeonol for $24 \mathrm{~h}$, the relative transcription levels of SIRT 3 (NPs with paeonol: 1.53 $\pm 0.41, P<0.01$; GDM pregnancies with paeonol: $1.22 \pm 0.16, P>0.05)$ and SIRT4 (NPs with paeonol: $2.18 \pm 0.63, P<0.01$; GDM pregnancies with paeonol: $1.74 \pm 0.35, P>0.05)$ markedly increased in ECFCs of NPs but not in those of GDM pregnancies. There were no significant differences in $\mathrm{NAD}^{+}$concentration (NPs with paeonol: $1.21 \pm 0.34, P>0.05$; GDM pregnancies with 
a

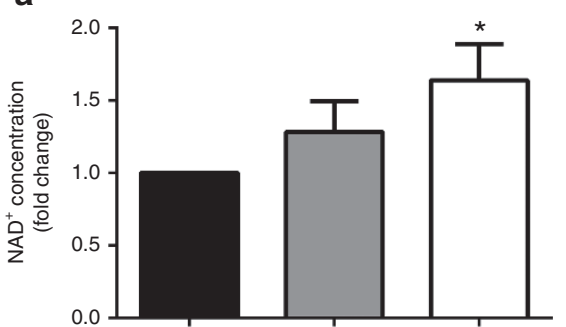

d

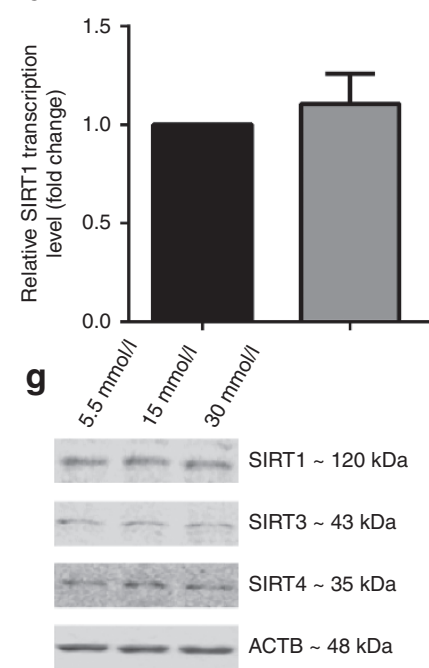

b

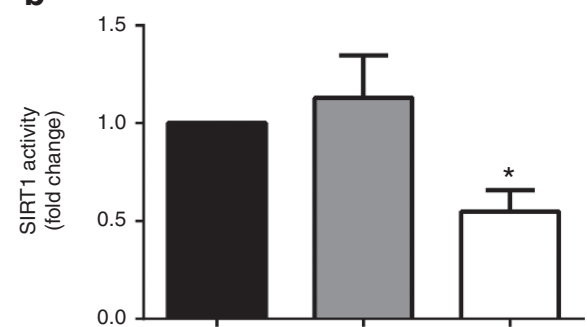

e

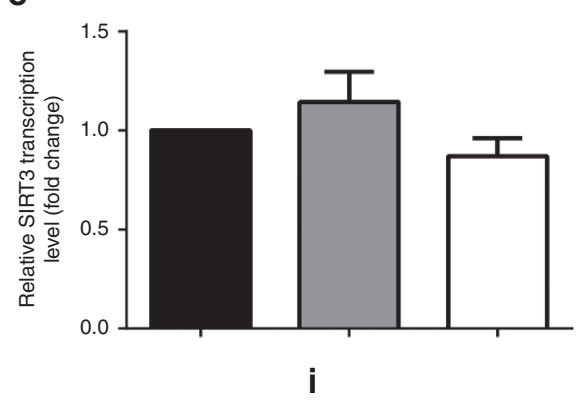

C

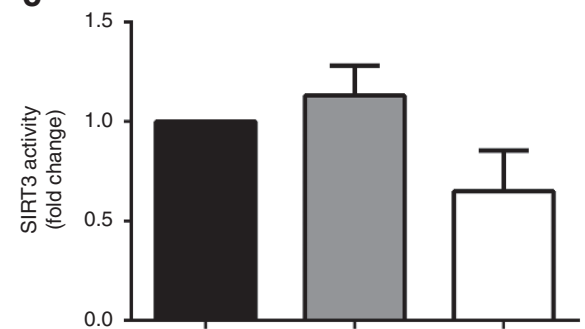

f

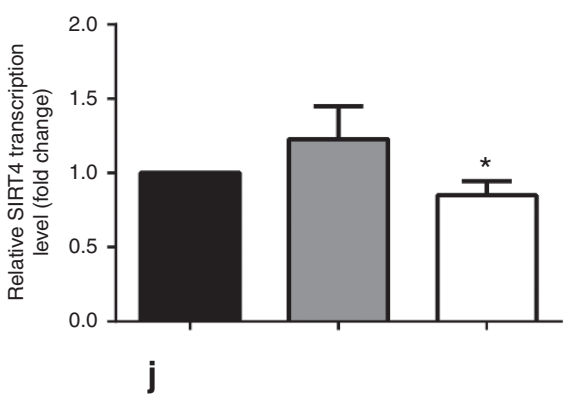

h

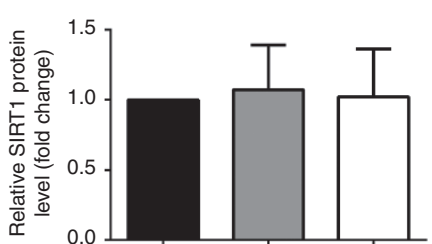

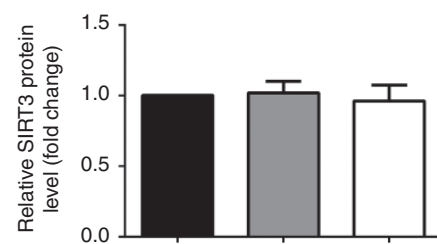

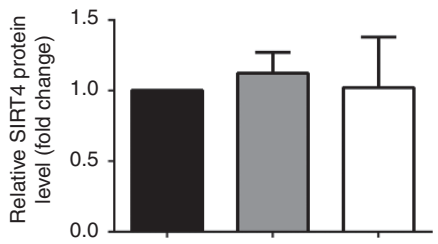

Figure 3. Alterations of $\mathrm{NAD}^{+}$and sirtuins (SIRTs) in endothelial colony-forming cells (ECFCs) from uncomplicated pregnancies (NPs; black) treated with 15-mM (gray) and 30-mM (white) glucose. (a) NAD concentration, (b) SIRT1 activity, (c) SIRT3 activity, relative transcription levels of (d) SIRT1, (e) SIRT3, and (f) SIRT4 in ECFCs from NPs after incubation with 15-mM or 30-mM glucose compared with standard culture medium (5.5 mM). (g) Representative immunoblots and relative protein levels of (h) SIRT1, (i) SIRT3, and (j) SIRT4 of ECFCs from NPs treated with 5.5-, 15-, or 30-mM glucose with no significant differences. $n=5$. Data are shown as mean fold changes \pm SD of 15- or 30-mM-glucose-treated ECFCs compared with 5.5-mM-glucose-treated ECFCs (control $=1) .{ }^{*} P<0.05$ when compared with 5.5 -mM-glucose-treated ECFCs.

paeonol: $1.19 \pm 0.56, P>0.05$ ), SIRT1 activity (NPs with paeonol: $1.39 \pm 0.50, P>0.05$; GDM pregnancies with paeonol: $1.56 \pm 0.52, P>0.05$ ), SIRT3 activity (NPs with paeonol: $0.99 \pm 0.43, P>0.05$; GDM pregnancies with paeonol: $1.36 \pm 0.41, P>0.05$ ), or SIRT1 transcription level (NPs with paeonol: $2.48 \pm 0.13, P>0.05$; GDM pregnancies with paeonol: $0.86 \pm 0.17, P>0.05$ ) (Figure 5).

After treatment with $1-\mu \mathrm{M}$ RSV for $24 \mathrm{~h}$, the $\mathrm{NAD}^{+}$concentration significantly increased in ECFCs from NPs but not in those from GDM pregnancies (NPs with RSV: $1.48 \pm 0.24$, $P<0.05$; GDM pregnancies with RSV: $1.38 \pm 0.26, P>0.05$ ), and SIRT1 activity was stimulated in both NPs and GDM pregnancies (NPs with RSV: $1.68 \pm 0.22, P<0.05$; GDM pregnancies with RSV: $2.04 \pm 0.64, P<0.01)$. SIRT3 activity markedly increased only in GDM pregnancies (NPs with RSV: $1.00 \pm 0.40$, $P>0.05$; GDM pregnancies with RSV: $1.68 \pm 0.23, P<0.05)$. The relative transcription level of SIRT1 (NPs with RSV: $3.57 \pm 0.45$, $P<0.01$; GDM pregnancies with RSV: $1.37 \pm 0.20, P>0.05)$ and SIRT3 (NPs with RSV: $1.54 \pm 0.57, P<0.01$; GDM pregnancies with RSV: $1.14 \pm 0.18, P>0.05)$ increased significantly only in ECFCs of NPs but not in those of GDM pregnancies, while there were no changes in SIRT4 transcription levels (NPs with RSV: $1.72 \pm 0.81, P>0.05$; GDM pregnancies with RSV:
$1.08 \pm 0.19, P>0.05)$. Paeonol and RSV both did not significantly change protein levels of SIRT1, SIRT3, and SIRT4 in NPs and GDM pregnancies $(P>0.05)$ (Figure 5).

\section{Effect of SIRT Activators on HUVECs}

Control group represents cells mock treated with ethanol from each patient group. Treatment with $50-\mu \mathrm{M}$ paeonol for $24 \mathrm{~h}$ increased the relative transcription level of SIRT4 (NPs with paeonol: $1.42 \pm 0.33, P<0.05$; GDM pregnancies with paeonol: $1.37 \pm 0.36, P<0.05)$ in both NPs and GDM pregnancies (Figure 6). However, paeonol did not significantly impact $\mathrm{NAD}^{+}$concentration (NPs with paeonol: $1.31 \pm 0.23, P>0.05$; GDM pregnancies with paeonol: $1.13 \pm 0.12, P>0.05)$, SIRT1 activity (NPs with paeonol: $1.10 \pm 0.15, P>0.05$; GDM pregnancies with paeonol: $1.07 \pm 0.52, P>0.05)$, SIRT3 activity (NPs with paeonol: $0.98 \pm 0.25, P>0.05$; GDM pregnancies with paeonol: $0.94 \pm 0.20, P>0.05)$, and the relative transcription levels of SIRT1 (NPs with paeonol: $1.15 \pm 0.38, P>0.05$; GDM pregnancies with paeonol: $1.12 \pm 0.38, P>0.05)$ and SIRT3 (NPs with paeonol: $1.22 \pm 0.61, P>0.05$; GDM pregnancies with paeonol: $1.03 \pm 0.14, P>0.05$ )

After treatment with $1-\mu \mathrm{M}$ RSV for $24 \mathrm{~h}$, the $\mathrm{NAD}^{+}$concentration increased in NPs (NPs with RSV: $1.42 \pm 0.31, P<0.01$; 


\section{Articles Guiet al.}

a

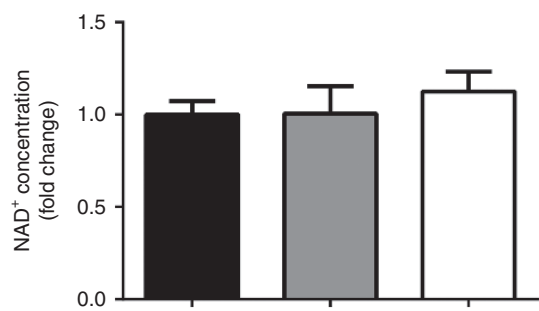

d

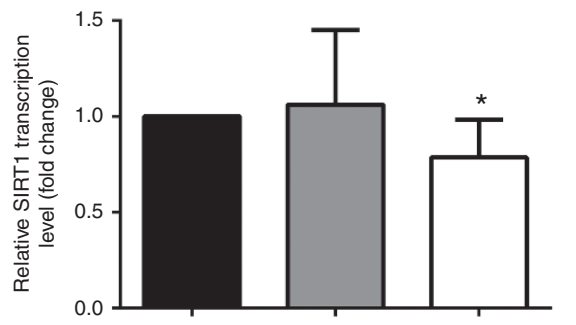

g

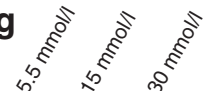

b

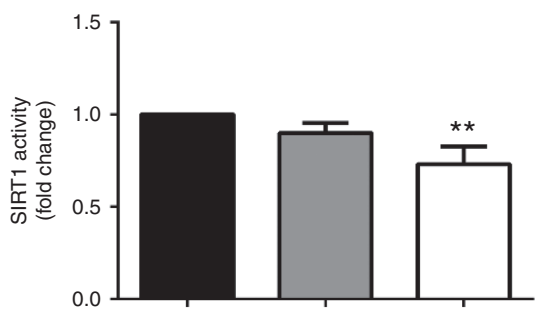

e

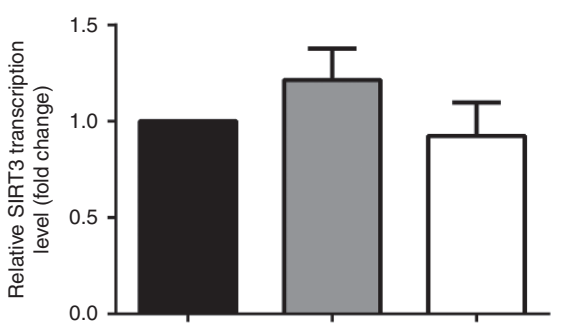

i
C

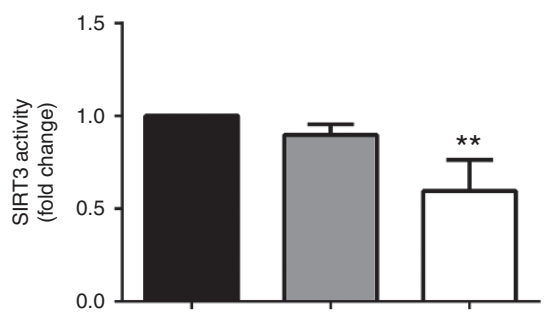

f

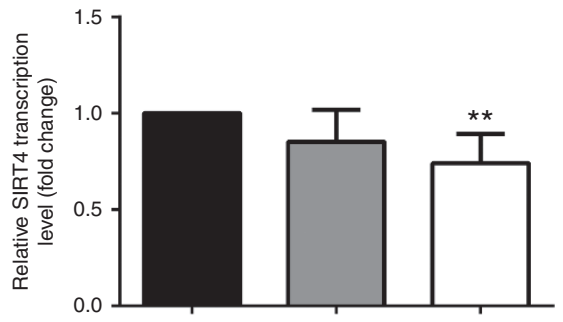

j
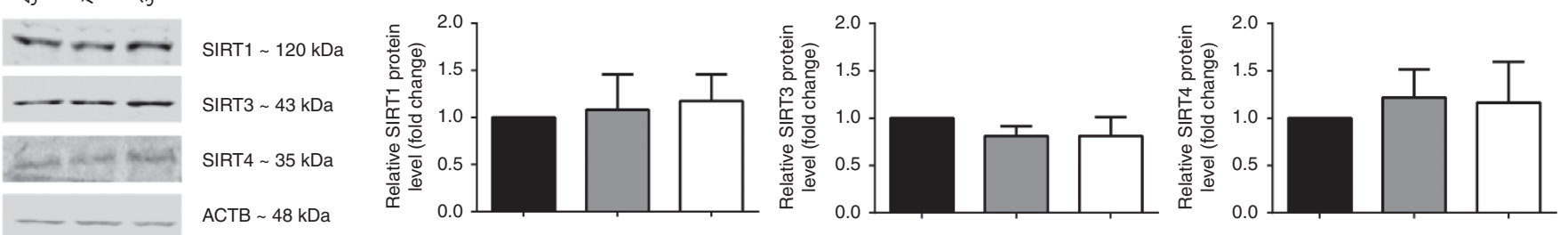

Figure 4. Alterations of $\mathrm{NAD}^{+}$and sirtuins (SIRTs) in human umbilical vein endothelial cells (HUVECs) from uncomplicated pregnancies (NPs; black) treated with 15-mM (gray) and 30-mM (white) glucose. (a) NAD ${ }^{+}$concentration, (b) SIRT1 activity, (c) SIRT3 activity, relative transcription levels of (d) SIRT1, (e) SIRT3, and (f) SIRT4 in HUVECs of NPs after incubation with 15-mM or 30-mM glucose compared with standard culture medium (5.5 mM). (g) Representative immunoblots and relative protein levels of (h) SIRT1, (i) SIRT3, and (j) SIRT4 of ECFCs from NPs treated with 5.5-, 15--, or 30-mM glucose. No significant alterations in protein levels were detected. $(n=5)$. Data are shown as mean fold changes \pm SD of 15- or 30-mM-glucose-treated HUVECs compared with 5.5-mM-glucose-treated HUVECs (control = 1). ${ }^{*} P<0.05,{ }^{* *} P<0.01$ when compared with 5.5-mM-glucose-treated ECFCs.

GDM pregnancies with RSV: $1.20 \pm 0.15, P>0.05)$. SIRT1 activity (NPs with RSV: $1.61 \pm 0.25, P<0.01$; GDM pregnancies with RSV: $1.66 \pm 0.14, P<0.01$ ) and SIRT3 activity (NPs with RSV: $1.52 \pm 0.21, P<0.01$; GDM pregnancies with RSV: $1.48 \pm 0.38$, $P<0.01)$ were markedly stimulated in HUVECs from NPs and GDM pregnancies. The relative transcription level of SIRT1 (NPs with RSV: $1.49 \pm 0.97, P<0.01$; GDM pregnancies with RSV: $1.40 \pm 0.64, P<0.05)$ was higher in NPs and GDM pregnancies, while the relative transcription levels of SIRT3 (NPs with RSV: $1.11 \pm 0.52, P>0.05$; GDM pregnancies with RSV: $0.96 \pm 0.28, P>0.05)$ and SIRT4 (NPs with RSV: $1.32 \pm 0.22$, $P>0.05$; GDM pregnancies with RSV: $1.13 \pm 0.33, P>0.05)$ were not significantly different. Paeonol and RSV both did not significantly change protein levels of SIRT1, SIRT3, and SIRT4 in NPs and GDM pregnancies $(P>0.05)$ (Figure 6).

\section{DISCUSSION}

In the 1980s, Barker (1) proposed the concept of fetal programming as a possible cause of cardiovascular morbidity and mortality in adulthood. In line with this theory, recent clinical trials have shown that normalization of blood glucose failed to improve cardiovascular morbidity in the diabetic population $(20,21)$.
Furthermore, increased levels of circulating cellular adhesion molecules, putative biomarkers for early subclinical stages of atherosclerosis and diabetes, have been found in offspring exposed to GDM and preexisting maternal diabetes (22). The persistence of hyperglycemic stress despite glucose normalization has been coined "hyperglycemic memory" (23). This emerging concept of a metabolic memory may explain why cardiovascular complications and other sequelae progress in the offspring of GDM pregnancies despite normoglycemia. However, it is not clear how fetal hyperglycemia translates into morbidity and mortality during later life. Therefore, we aimed to study the effect of GDM on SIRTs as potential mediators of altered vascular homeostasis.

In the present study, we show that SIRT levels of ECFCs isolated from cord blood and HUVECs isolated from cord are altered in pregnancies complicated by GDM pregnancies compared with NPs. The activity and expression levels of SIRTs were lower in both GDM-ECFCs and GDM-HUVECs compared with NPs. Comparable effects were also found after exposure of ECFCs and HUVECs from NPs to hyperglycemia in vitro. Furthermore, SIRT reduction was more pronounced in GDMECFCs compared with GDM-HUVECs. We also found that the SIRT activator RSV could significantly enhance the expression 
a

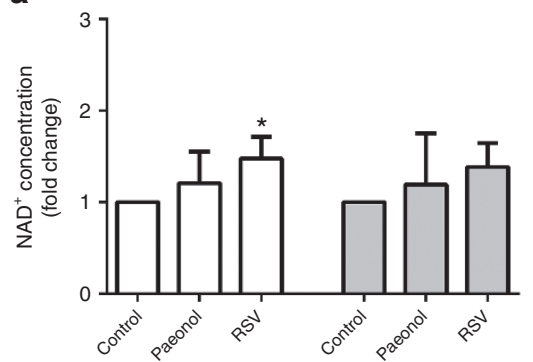

d

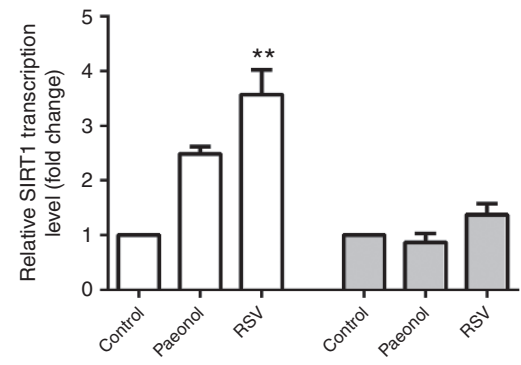

b

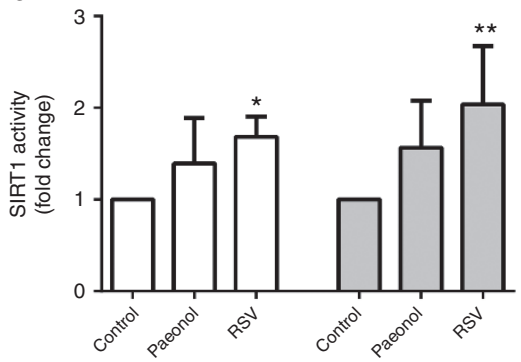

e

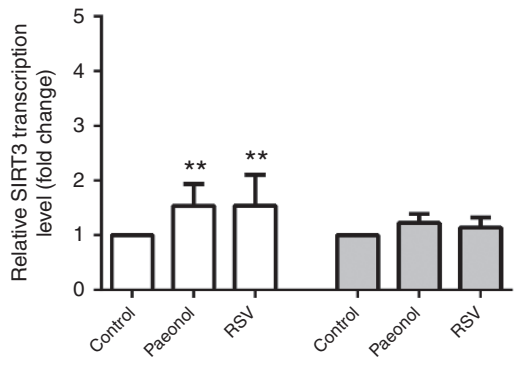

i

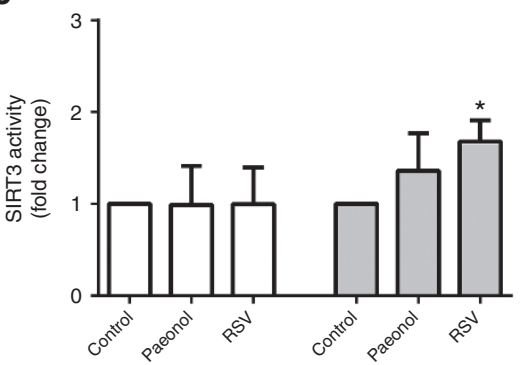

f

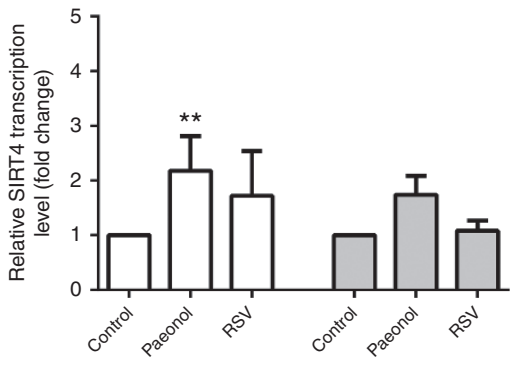

g

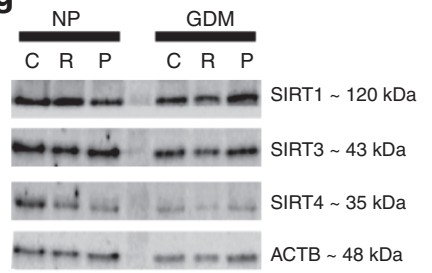

h

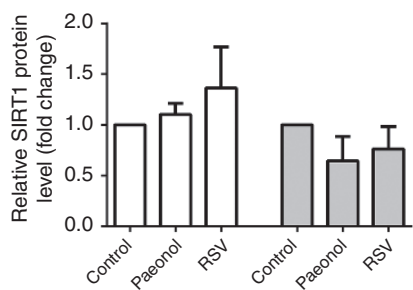

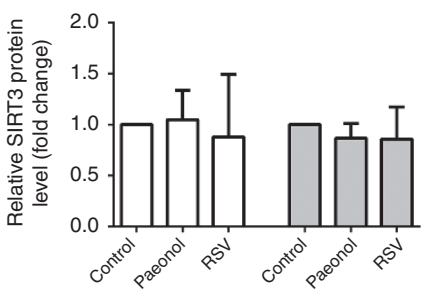

j

Figure 5. Effect of sirtuin (SIRT) activators on endothelial colony-forming cells (ECFCs) from gestational diabetes (GDM) pregnancies (gray) compared with uncomplicated pregnancies (NPs; white). ECFCs were incubated with $50-\mu \mathrm{M}$ paeonol resuspended in dimethylsulfoxide or 1- $\mu \mathrm{M}$ resveratrol (RSV) resuspended in ethanol for $24 \mathrm{~h}$ before collection. Control cells were mock treated with ethanol. (a) NAD+ concentration, (b) SIRT1 activity, (c) SIRT3 activity and relative transcription levels of (d) SIRT1, (e) SIRT3, and (f) SIRT4 in ECFCs of NPs and GDM pregnancies. (g) Representative immunoblots and relative protein levels of (h) SIRT1, (i) SIRT3, and (j) SIRT4 of ECFCs from NPs and GDM pregnancies treated with ethanol, RSV, or paeonol. No significant alterations in protein levels were detected. $(n=5)$. Data are presented as mean fold changes \pm SD of RSV- or paeonol-treated ECFCs as compared with mock-treated ECFCs (control $=1) .{ }^{*} P<0.05$ or ${ }^{* *} P<0.01$ when compared with control.

and activity of SIRTs in both ECFCs and HUVECs. However, the SIRT activator paeonol only had an effect in ECFCs.

The downregulation of SIRT1 observed in ECFCs from neonates with pregnancies complicated by GDM suggests that SIRT1 in circulating blood cells may serve as a novel biomarker, confirming results of a study by de Kreutzenberg et al. (8) in patients with insulin resistance and metabolic syndrome. Downregulation of SIRT1 has previously been described to be associated with accelerated senescence of EPCs in neonates underlining its function as a key regulator in vascular homeostasis during early life (24). In accordance with our results, Balestrieri et al. (10) observed decreased SIRT1 protein levels in EPCs from type 2 diabetic patients, especially those with poor glycemic control. Zheng et al. (25) showed that decreased levels of SIRT1 correlated with the cellular "memory" of hyperglycemic stress after glucose levels were normalized. Activation of SIRT1 by RSV or SIRT1 overexpression compromised the memory of hyperglycemic stress, whereas the inhibition of SIRT1 by SIRT1 siRNA increased sensitivity to high-glucose stress. SIRT1 has been reported to control endothelial angiogenic functions during postnatal vascular growth (26). Our previous findings of impaired functions, e.g., proliferation, migration, and angiogenesis of ECFCs from GDM pregnancies (18) could potentially result from lower SIRT activity. Thus, SIRTs seem to be promising candidate molecules for mediating the feature of "hyperglycemic memory".

In the present study, we found a downregulation of SIRT3 activity and SIRT3 and SIRT4 at mRNA level in GDM pregnancies. SIRT activators enhanced SIRT3 and SIRT4 mRNA expression. These results may imply metabolic rescue by the induction of SIRT1 activity. As previously described, SIRT1 activates peroxisome proliferator-activated receptor gamma coactivator 1-alpha (PGC1 $\alpha)(27)$, which is a regulator of mitochondrial biogenesis and function. PGC1 $\alpha$ integrates different cellular signals and regulates genes of energy metabolism, one of these genes is SIRT3. It is activated by PGC1 $\alpha$ binding to the estrogen-related receptor-binding element in the SIRT3 promotor region (28). Feldman et al. (29) hypothesized 
a

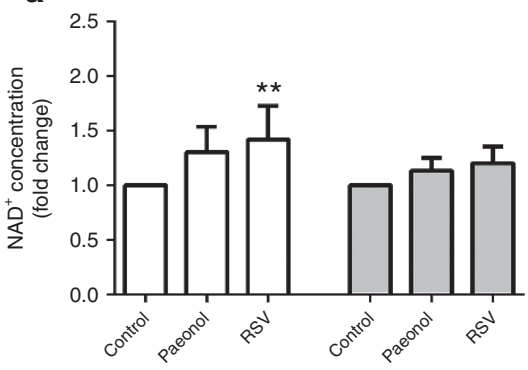

d

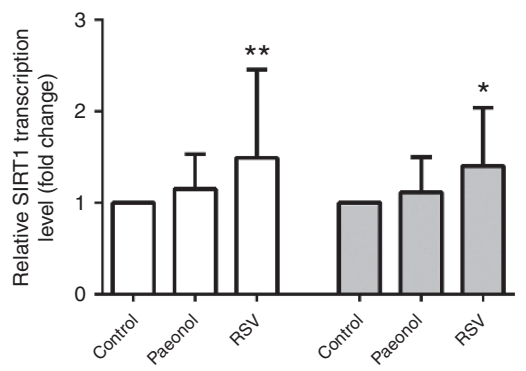

g

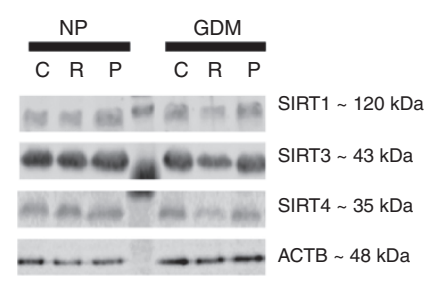

b

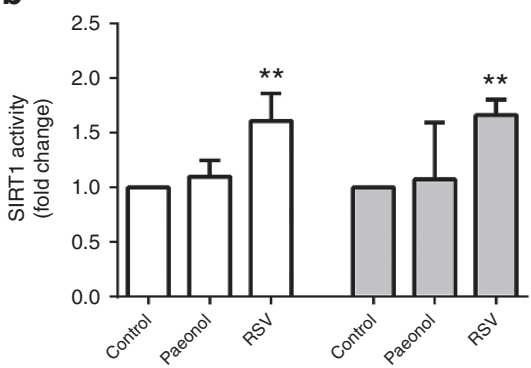

e

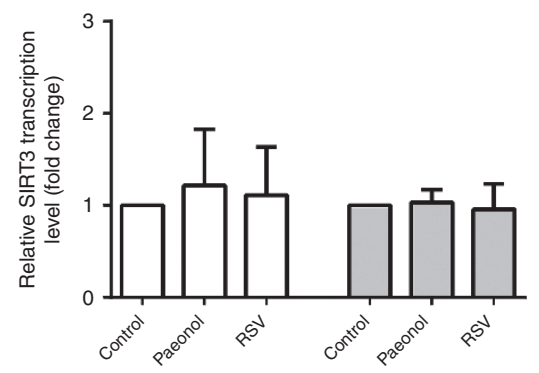

i c

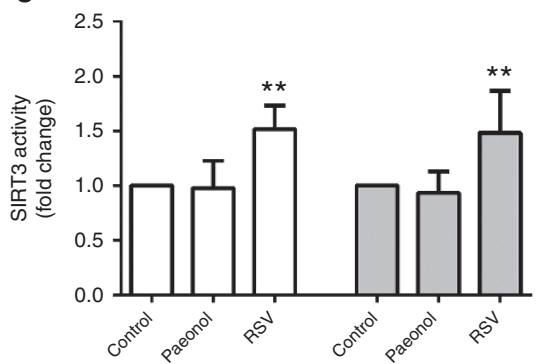

f

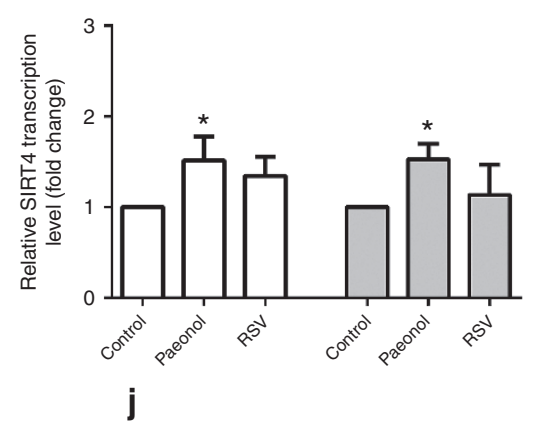

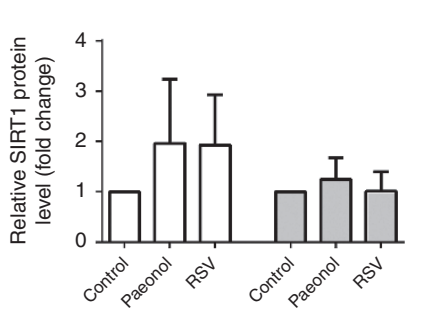
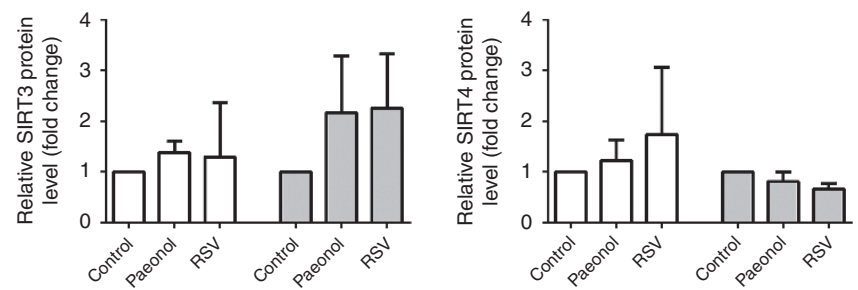

Figure 6. Effect of sirtuin (SIRT) activators on human umbilical vein endothelial cells (HUVECs) from gestational diabetes (GDM) pregnancies (gray) compared with uncomplicated pregnancies (NPs; white). HUVECs were incubated with $50-\mu \mathrm{M}$ paeonol resuspended in dimethylsulfoxide or $1-\mu \mathrm{M}$ resveratrol (RSV) resuspended in ethanol for $24 \mathrm{~h}$ before collection. Control cells were mock treated with ethanol. (a) NAD ${ }^{+}$concentration, (b) SIRT1 activity, (c) SIRT3 activity and relative transcription levels of (d) SIRT1, (e) SIRT3, and (f) SIRT4 in HUVECs of NPs and GDM pregnancies. (g) Representative immunoblots and relative protein levels of (h) SIRT1, (i) SIRT3, and (j) SIRT4 of HUVECs from NPs and GDM pregnancies treated with ethanol, RSV, or paeonol. No significant differences in protein levels were detected. $(n=5)$. Data are presented as mean fold changes \pm SD of RSV- or paeonol-treated HUVECs as compared with mock-treated HUVECs (control $=1) .{ }^{*} P<0.05$ or ${ }^{* *} P<0.01$ when compared with control.

that the PGC1 $\alpha$-dependent mitochondrial metabolic reprogramming is SIRT3 mediated. This crosstalk of the nutrientsensitive SIRT1 and the mitochondrial regulators PGC1 $\alpha$ and SIRT3 may be one of the reasons for altered metabolic regulation of GDM cells and later on in children of GDM pregnancies. Furthermore, SIRT3 reduction is associated with altered metabolic conditions like high-fat feeding or fasting. Huynh et al. (30) speculated that patients with type 2 diabetes show decreased SIRT3 levels due to an altered metabolic homeostasis. Our results seem to support this theory, even if we are not able to provide a molecular mechanism at this point.

Hyperglycemia has been associated with increased oxygen consumption, which is probably related to an increased production of reactive oxygen species (ROS) (31). SIRT3 plays a key role in regulating mitochondrial ROS homeostasis (32). Increased ROS production in vascular tissue under pathological conditions is the main cause of endothelial dysfunction. The SIRT activator RSV attenuated oxidative injury in endothelial cells through the regulation of mitochondrial ROS homeostasis, which, in part, was mediated through the activation of SIRT3 (33).

Data on the role of SIRT4 are limited, but inhibition is likely to accelerate the development of type 2 diabetes (34). In general, SIRT4 is associated with the regulation of energy metabolism. Recent data suggest that SIRT4 is a cellular, especially mitochondrial, lipoamidase. Only a few proteins are lipoylated, but they play critical roles in cellular metabolism and are targets of SIRT4 (35). One of those targets is pyruvate dehydrogenase, which is inhibited after SIRT4-dependent hydrolysis of the lipoamide. The reduction of SIRT4 in GDM cells in our study may serve to maintain pyruvate dehydrogenase activity (35) thus allowing for metabolism of glucose. SIRT4 is known to inhibit fatty acid oxidation via increasing levels of malonylCoA. If SIRT4 is reduced in GDM cells, fatty acid oxidation will be stimulated thus enhancing energy production. Furthermore, the efficiency of the adenosine triphosphate production is regulated via the adenosine diphosphate/triphosphate translocator ANT2, which not only acts as an adenosine 
diphosphate/triphosphate exchange transporter but as an uncoupler as well (36). Decreased SIRT4 levels would lead to a less efficient energy production. It is not clear if reduced SIRT4 levels are confined to ECFCs and HUVECs or are a general feature in many organs. We do not know which effect is the most important one for the dysregulation of SIRT4 in GDM pregnancies. Further work has to be performed on SIRT4 to get sufficient insight into its regulation.

To the best of our knowledge, this is the first study investigating the effect of the SIRT activators RSV and paeonol on GDM-ECFCs and GDM-HUVECs. The experiments presented here show that RSV could significantly enhance the expression and activity of SIRTs in both ECFCs and HUVECs from NPs and GDM pregnancies. However, the response to paeonol was heterogenous. In fact, paeonol only had a significant effect in ECFC. RSV is a polyphenolic compound from red grapes. It was the first compound described that can mimic the SIRTmediated effects of caloric restriction. Several different mechanisms for the action of RSV have been proposed (37). Gertz et al. (14) show a direct activation via interaction of RSV and the SIRT1 active site using crystal structure analysis. The authors also describe RSV as a strong activator of SIRT5 and an inhibitor of SIRT3. Other studies propose a mechanism via activation of the Akt pathway. Modulating the Akt pathway would explain the effects of RSV on the cell-cycle regulation in neurons (38). Further studies suggest an activation of the $5^{\prime}$ AMPactivated protein kinase (AMPK) pathway in a RSV/SIRT1/ SIRT3-dependent manner, but Desquiret-Dumas et al. (39) inhibited AMPK and could still measure RSV-induced mitochondrial biogenesis in liver cells. Mechanistic studies in endothelial cells propose an increase in vascular oxidative stress resistance induced by RSV via antioxidant, anti-apoptotic, and anti-inflammatory effects $(40,41)$ and a pathway that involves the activation of SIRT1 and the upregulation of antioxidant defense mechanisms by RSV to attenuate mitochondrial ROS production (42). Paeonol is a phenolic compound derived from peonies. Traditional Chinese medicine uses this compound alleviating various oxidative stress-related diseases. Jamal et al (15) suggest a beneficial role of paeonol on premature senescence in a SIRT1-dependent manner. However, the mechanism of action for this compound is still unknown.

SIRT1 activation is a promising new therapeutic approach for treating diseases of aging such as type 2 diabetes (43). SIRT1 activation by RSV improves vascular function by increasing NO availability and attenuates dyslipidemia and obesity-induced metabolic alterations in human subjects (44-46). Paeonol can alleviate the oxidative stress-related cardiovascular diseases predominantly through the modulation of endogenous antioxidants and reduction of free radical formation $(47,48)$.

Two different endothelial cell types-ECFCs and HUVECs-were used in this study. They are the first vascular cells of the fetus that are exposed to aberrant conditions and an adverse metabolic milieu during pregnancy. ECFCs represent progenitor cells, possessing the ability to differentiate into mature endothelial cells and to repair damaged endothelium. In contrast, HUVECs are mature endothelial cells which may explain an increased vulnerability of the latter, which is reflected in more pronounced changes of SIRT1 (33). At the same time, this finding also supports the hypothesis of "hyperglycemic memory”. As ECFCs are responsible for controlling vascular repair and development of the vascular system, they may mediate the "hyperglycemic memory" and altered SIRT expression of ECFCs might be another sign of endothelial cell dysfunction leading to reduced vessel repair capacity.

A few studies compared functional and molecular readouts in these two cell types and showed heterogeneous responses in many cases. Sipos et al. (49) describe the ability of human fetal ECFCs, but not human fetal endothelial cells, to transmigrate to the maternal bloodstream of the uterus and then home to locations of maternal uterine vasculogenesis underlining profound functional differences between these cell types. While the response of ECFCs to shear stress was comparable with HUVECs and human umbilical artery endothelial cells (50), a differential response of ECFCs compared with HUVECs was detected on electrospun scaffolds with distinct microfiber diameters (51). In 3D assays, ECFCs induced more robust sprouting vascular structures than HUVECs possibly induced by a stronger phosphorylation of VEGFR2 and its downstream signaling (52). The experiments presented here show that ECFCs but not HUVECs respond to paeonol. Own, unpublished data indicate a strong functional response of ECFCs to vitamin D, while HUVECs did not change their proliferation, migration, and angiogenic behavior. These data are in support of the theory of a differential sensitivity of these cells to the same stimuli.

Our patients were recruited at admission to Labor \& Delivery. To avoid the impact of other factors than GDM on our results, we matched the two groups as best as possible according to gestational age, maternal age, and pre-pregnancy BMI. This resulted in an average BMI in the overweight range also in the group of women with NPs and is therefore higher than we would expect it to be in the general population of women with NPs.

\section{Conclusion}

In summary, we have shown that GDM and in vitro hyperglycemia induced a reduction of SIRTs in fetal ECFCs and HUVECs. These findings might reflect one underlying mechanism resulting in metabolic and vascular diseases in the offspring of GDM pregnancies. SIRT activators, paeonol and RSV, improved the function of SIRTs in ECFCs, which opens new perspectives for the prevention of adverse long-term effects of a diabetic intrauterine environment. Our findings give new insights into understanding and controlling the increased risk of cardiovascular diseases and other long-term sequelae in individuals originating from pregnancies complicated by GDM. However, further research is needed to explore the underlying cellular and molecular mechanisms and the potential of SIRT activators to improve vascular function.

\section{METHODS}

\section{Patients}

Ten women with NPs and ten women with GDM pregnancies were recruited for this study and matched for gestational age, maternal age, 
and pre-pregnancy BMI. The diagnostic standard for GDM was based on the criteria defined by the "International Association of Diabetes and Pregnancy Study Groups". All women had an oral glucose tolerance test at 24-28 wk of gestation, singleton pregnancies, and normal blood pressure during pregnancy. In addition, all women had no evidence of illnesses known to affect glucose metabolism, e.g., no clinical history of preexisting diabetes, renal, hypertensive, or other vascular disease, and none were on medication known to affect glucose metabolism. All patients were nonsmokers by self-report. All participants in the study gave written informed consent. The Ethical Committee at Hannover Medical School approved this study.

\section{Isolation of ECFCs and HUVECs}

Umbilical cord and umbilical cord venous blood were collected immediately after delivery. ECFCs from cord blood were isolated as previously described (53). The culture medium (complete endothelial growth medium, EGM-2) was endothelial basal medium 2 (Lonza, Walkersville, MD) with 10\% fetal calf serum (Biochrom AG, Berlin, Germany) and $1 \%$ penicillin/streptomycin (Biochrom AG) supplemented with EGM-2 SingleQuots Kit (Lonza). The medium was changed daily for $10 \mathrm{~d}$ and then every other day until the first passage. Colonies of ECFCs appeared between 5 and $30 \mathrm{~d}$ of cultivation and were identified as circumscribed monolayers of more than 50 rapidly proliferating, cobblestone-appearing cells. ECFCs were used at $80-90 \%$ confluence at passage 4 or 5 .

HUVECs were harvested from fresh human umbilical cord vessels within 1-5 days of delivery. Briefly, umbilical veins were cannulated with blunt needles and rinsed with PBS. Then type I collagenase (180 units/mg, GIBCO, Invitrogen, CA) was infused. Segments were clamped at both ends and placed in an incubator at $37^{\circ} \mathrm{C}$ for $30 \mathrm{~min}$. Segments were rinsed with PBS, and the cell suspension was centrifuged. The cell pellet was resuspended and plated in tissue culture flasks in endothelial cell growth medium with supplemental mix (Promocell, Heidelberg, Germany) containing 10\% fetal calf serum and 1\% penicillin/streptomycin. Passage 5 was used for further experimentation.

Immunofluorescence and flow cytometry were used to confirm the ECFC phenotype as previously described (53). Purity of the HUVEC cultures was determined by flow cytometric analysis (Partec $\mathrm{GmbH}$, Münster, Germany) using the fluorescein-isothiocyanate-conjugated monoclonal anti-human-endothelial cell antibody CD31 and the R-phycoerythrin-conjugated monoclonal anti-human-fibroblast antibody CD90 (clone WM59 and 5E10; Dako Cytomation GmbH, Hamburg, Germany).

\section{Treatment Conditions}

Hyperglycemia treatment included a 7-day exposure of NP ECFCs or HUVECs with supplemental D-glucose (Sigma-Aldrich, Seelze, Germany) at 15.0 or $30.0 \mathrm{mM}$ added to complete EGM-2 medium (5.5 mM glucose, standard medium). ECFCs and HUVECs were incubated with $50 \mu \mathrm{M}$ paeonol (Sigma-Aldrich, St. Louis, MO) resuspended in dimethylsulfoxide (Sigma-Aldrich) or $1 \mu \mathrm{M}$ RSV (Enzo Life Sciences, NY) resuspended in ethanol for $24 \mathrm{~h}$ before collection. The concentrations used of RSV and paeonol were chosen according to published literature, in which a relevant or optimal dose has not been established by human studies and will vary depending on the effect being studied $(14,15)$. Control cells were mock treated with ethanol.

\section{NAD ${ }^{+}$Measurement}

Cells were resuspended in HEPES buffer (Sigma-Aldrich) with a concentration of 500,000 cells $/ \mathrm{ml}$. The measurement was performed as previously described (54). The absorption was measured at $340 \mathrm{~nm}$ using the Infinite M200pro spectrophotometer (Tecan, Maennedorf, Switzerland).

\section{SIRT Activity}

Maximal SIRT activity was determined as per the manufacturer's recommendations using the SIRT1 Fluorometric Drug Discovery Kit BML-AK555 (Enzo Life Sciences, Farmingdale, NY) and the SIRT3 Fluorimetric Drug Discovery Kit BML-AK557 (Enzo Life Sciences). Samples (cells in HEPES buffer at a concentration of 500,000 cells $/ \mathrm{ml}$ ) were diluted at a ratio of 1:3. The signal was measured with the Infinite
M200pro spectrophotometer (Tecan) at an excitation of $365 \mathrm{~nm}$ and at an emission of $465 \mathrm{~nm}$.

\section{Western Blot Analysis}

After trypsinization, cells were lysed in $3 \times$ Laemmli buffer (Tris $\mathrm{HCl}$ $2 \mathrm{M} 4.725 \mathrm{ml}$, glycerol $20 \% 7.5 \mathrm{ml}$, sodium dodecyl sulfate $10 \% 30 \mathrm{ml}$, 2-mercaptoethanol $4.5 \mathrm{ml}, \mathrm{H}_{2} \mathrm{O} 3.275 \mathrm{ml}$, bromophenolblue $500 \mu \mathrm{g}$, $\mathrm{pH}=6.8,20 \mu \mathrm{l} / 100,000$ cells). Ten microliter of protein were separated on a sodium dodecyl sulfate containing $10 \%$ polyacrylamide gel, then transferred to nitrocellulose membranes, and incubated with blocking buffer (Odyssey Li-Cor Biosciences, Lincoln, NE) containing 5\% bovine serum albumin (GERBU Biotechnik GmbH, Heidelberg, Germany) for $1 \mathrm{~h}$ at room temperature. The membranes were subsequently incubated overnight at $4{ }^{\circ} \mathrm{C}$ with primary antibodies (SIRT 1 diluted 1:250, SIRT 3 diluted 1:250, $\beta$-actin as internal control diluted 1:2,000, all rabbit polyclonal, Millipore, Temecula, CA), washed in Tris Buffered Saline with 0.1\% Tween-20 (TBS-T) $4 \times$ for $5 \mathrm{~min}$, and then incubated with secondary antibody (goat antirabbit IRDye $800 \mathrm{CW}, 0.5 \mathrm{mg}, 1: 20000$, Odyssey Li-Cor Biosciences) for $1 \mathrm{~h}$. After washing with TBS-T $3 \times$ for $5 \mathrm{~min}$ and TBS $1 \times$ for $5 \mathrm{~min}$, Odyssey Fc Dual-Mode Imaging System (Li-Cor Biosciences) was used to detect the bands and estimate the density of proteins. For the analysis of $\beta$-actin, the membranes were stripped and reprobed with anti- $\beta$-actin antibody to account for protein loading variations.

\section{RNA Isolation and Real-Time RT-PCR}

Total RNA was isolated using RNeasy Micro Kit (Qiagen, Hilden, Germany) according to the manufacturer's instruction. One microgram of RNA was reverse-transcribed using Omniscript reverse transcription kit (Qiagen). SYBR Green PCR Master Mix (Life technologies, Grand Island, NY) was used for real-time RT-PCR according to the manufacturer's instructions in 7900HT Fast Real Time PCR System (Applied Biosystems, Foster City, CA). cDNA templates were diluted $1: 30$. The program involved initially $15 \mathrm{~min}$ at $95^{\circ} \mathrm{C}$, at $60^{\circ} \mathrm{C}$ for $30 \mathrm{~s}$, at $72^{\circ} \mathrm{C}$ for $30 \mathrm{~s}$ for 45 cycles afterwards. Relative quantification of the signals was performed by normalizing the signals of different genes with the average of all internal genes. Signal intensities in the control lanes were arbitrarily assigned a value of 1.0. The primer sequences (forward/reverse, Eurofins, Brussels, Belgium) used were as follows: SIRT 1, 5' - CAA CTT GTA CGA CGA AGA C-3'/ 5' -TCA TCA CCG AAC AGA AGG-3'; SIRT 3, 5'- CAG TCT GCC AAA GAC CCT TC-3'/ 5' - AAA TCA ACC ACA TGC AGC AA-3'; SIRT 4, 5'-GCT GTG AGA GAA TGA AGA TGA GC-3'/5' - CTT GGA AAG GGT GAT GAA GCG-3'; $\beta$-actin, $5^{\prime}$-TTC CTG GGC ATG GAG TC-3'/ $5^{\prime}$ - CAG GTC TTT GCG GAT GTC-3'; hHPRT 1 , $5^{\prime}$-GCT GAC CTG CTG GAT TAC-3'/ 5'-TGC GAC CTT GAC CAT CTT-3'; GAPDH, 5'-TGC ACC ACC AAC TGC TTA GC-3'/ 5'-GGC ATG GAC TGT GGT CAT GAG-3'.

\section{Statistical Analysis}

Demographic data were analyzed using unpaired $t$ test and are expressed as means and SD or percentages. Differences in experimental data between two groups were compared by paired Wilcoxonsigned rank test for repeated measurements. Datasets with three or more groups were statistically analyzed by Kruskal-Wallis test and data are presented as mean fold changes and SD compared with controls. Controls were NPs, 5.5-mM, or mock-treated NPs or GDM samples as appropriate. All calculations were performed using Prism 6 software package (GraphPad Software, La Jolla, CA) with $P$ less than 0.05 considered statistically significant.

\section{ACKNOWLEDGMENTS}

We are grateful to all study participants and the staff of the Division of Obstetrics at Hannover Medical School for invaluable assistance in obtaining samples.

\section{STATEMENT OF FINANCIAL SUPPORT}

The study was supported by funding from the German Diabetes Association (Deutsche Diabetes Gesellschaft), Vitaflo Ltd. (Liverpool, UK), and a scholarship from the Chinese Scholarship Council (J.G.).

Disclosure: The authors declare that they have no competing interests. 


\section{REFERENCES}

1. Barker DJ. Intrauterine programming of adult disease. Mol Med Today 1995;1:418-23.

2. Durnwald C, Landon M. Fetal links to chronic disease: the role of gestational diabetes mellitus. Am J Perinatol 2013;30:343-6.

3. Desai M, Beall M, Ross MG. Developmental origins of obesity: programmed adipogenesis. Curr Diab Rep 2013;13:27-33.

4. Guarente L. Franklin H. Epstein Lecture: Sirtuins, aging, and medicine. N Engl J Med 2011;364:2235-44.

5. Nogueiras R, Habegger KM, Chaudhary N, et al. Sirtuin 1 and sirtuin 3: physiological modulators of metabolism. Physiol Rev 2012;92:1479-514.

6. Imai S, Armstrong CM, Kaeberlein M, Guarente L. Transcriptional silencing and longevity protein Sir2 is an NAD-dependent histone deacetylase. Nature 2000;403:795-800.

7. Balestrieri ML, Servillo L, Esposito A, et al. Poor glycaemic control in type 2 diabetes patients reduces endothelial progenitor cell number by influencing SIRT1 signalling via platelet-activating factor receptor activation. Diabetologia 2013;56:162-72.

8. de Kreutzenberg SV, Ceolotto G, Papparella I, et al. Downregulation of the longevity-associated protein sirtuin 1 in insulin resistance and metabolic syndrome: potential biochemical mechanisms. Diabetes 2010;59: 1006-15.

9. Mattagajasingh I, Kim CS, Naqvi A, et al. SIRT1 promotes endotheliumdependent vascular relaxation by activating endothelial nitric oxide synthase. Proc Natl Acad Sci U S A 2007;104:14855-60.

10. Balestrieri ML, Rienzo M, Felice F, et al. High glucose downregulates endothelial progenitor cell number via SIRT1. Biochim Biophys Acta 2008;1784:936-45.

11. Zhang J, Zhang X, Li H, et al. Hyperglycaemia exerts deleterious effects on late endothelial progenitor cell secretion actions. Diab Vasc Dis Res 2013;10:49-56

12. Zhang QJ, Wang Z, Chen HZ, et al. Endothelium-specific overexpression of class III deacetylase SIRT1 decreases atherosclerosis in apolipoprotein E-deficient mice. Cardiovasc Res 2008;80:191-9.

13. Zhou S, Chen HZ, Wan YZ, et al. Repression of P66Shc expression by SIRT1 contributes to the prevention of hyperglycemia-induced endothelial dysfunction. Circ Res 2011;109:639-48.

14. Gertz M, Nguyen GT, Fischer F, et al. A molecular mechanism for direct sirtuin activation by resveratrol. PLoS One 2012;7:e49761.

15. Jamal J, Mustafa MR, Wong PF. Paeonol protects against premature senescence in endothelial cells by modulating Sirtuin 1 pathway. J Ethnopharmacol 2014;154:428-36.

16. Kao CL, Chen LK, Chang YL, et al. Resveratrol protects human endothelium from $\mathrm{H}(2) \mathrm{O}(2)$-induced oxidative stress and senescence via SirT1 activation. J Atheroscler Thromb 2010;17:970-9.

17. Povsic TJ, Goldschmidt-Clermont PJ. Endothelial progenitor cells: markers of vascular reparative capacity. Ther Adv Cardiovasc Dis 2008;2: 199-213.

18. Gui J, Rohrbach A, Borns K, et al. Vitamin D rescues dysfunction of fetal endothelial colony forming cells from individuals with gestational diabetes. Placenta 2015;36:410-8.

19. von Versen-Höynck F, Brodowski L, Dechend R, Myerski AC, Hubel CA. Vitamin D antagonizes negative effects of preeclampsia on fetal endothelial colony forming cell number and function. PLoS One 2014;9:e98990.

20. Patel A, MacMahon S, Chalmers J, et al. Intensive blood glucose control and vascular outcomes in patients with type 2 diabetes. N Engl J Med 2008;358:2560-72.

21. Gerstein HC, Miller ME, Byington RP, et al. Effects of intensive glucose lowering in type 2 diabetes. N Engl J Med 2008;358:2545-59.

22. West NA, Crume TL, Maligie MA, Dabelea D. Cardiovascular risk factors in children exposed to maternal diabetes in utero. Diabetologia 2011;54:504-7.

23. Ceriello A. Hypothesis: the "metabolic memory", the new challenge of diabetes. Diabetes Res Clin Pract 2009;86:Suppl 1:S2-6.

24. Vassallo PF, Simoncini S, Ligi I, et al. Accelerated senescence of cord blood endothelial progenitor cells in premature neonates is driven by SIRT1 decreased expression. Blood 2014;123:2116-26.
25. Zheng Z, Chen H, Li J, et al. Sirtuin 1-mediated cellular metabolic memory of high glucose via the LKB1/AMPK/ROS pathway and therapeutic effects of metformin. Diabetes 2012;61:217-28.

26. Potente M, Ghaeni L, Baldessari D, et al. SIRT1 controls endothelial angiogenic functions during vascular growth. Genes Dev 2007;21:2644-58.

27. Noriega LG, Feige JN, Canto C, et al. CREB and ChREBP oppositely regulate SIRT1 expression in response to energy availability. EMBO Rep 2011;12:1069-76.

28. Kong X, Wang R, Xue Y, et al. Sirtuin 3, a new target of PGC-1alpha, plays an important role in the suppression of ROS and mitochondrial biogenesis. PLoS One 2010;5:e11707.

29. Feldman JL, Dittenhafer-Reed KE, Denu JM. Sirtuin catalysis and regulation. J Biol Chem 2012;287:42419-27.

30. Huynh FK, Hershberger KA, Hirschey MD. Targeting sirtuins for the treatment of diabetes. Diabetes Manag (Lond) 2013;3:245-57.

31. Dabelea D, Hanson RL, Lindsay RS, et al. Intrauterine exposure to diabetes conveys risks for type 2 diabetes and obesity: a study of discordant sibships. Diabetes 2000;49:2208-11.

32. Tseng AH, Shieh SS, Wang DL. SIRT3 deacetylates FOXO3 to protect mitochondria against oxidative damage. Free Radic Biol Med 2013;63:222-34.

33. Zhou X, Chen M, Zeng X, et al. Resveratrol regulates mitochondrial reactive oxygen species homeostasis through Sirt3 signaling pathway in human vascular endothelial cells. Cell Death Dis 2014;5:e1576.

34. Mahlknecht U, Voelter-Mahlknecht S. Fluorescence in situ hybridization and chromosomal organization of the sirtuin 4 gene (Sirt4) in the mouse. Biochem Biophys Res Commun 2009;382:685-90.

35. Mathias RA, Greco TM, Oberstein A, et al. Sirtuin 4 is a lipoamidase regulating pyruvate dehydrogenase complex activity. Cell 2014;159:1615-25.

36. Ho L, Titus AS, Banerjee KK, et al. SIRT4 regulates ATP homeostasis and mediates a retrograde signaling via AMPK. Aging (Albany NY) 2013;5:835-49.

37. Pallàs M, Casadesús G, Smith MA, et al. Resveratrol and neurodegenerative diseases: activation of SIRT1 as the potential pathway towards neuroprotection. Curr Neurovasc Res 2009;6:70-81.

38. Forero DA, Casadesus G, Perry G, Arboleda H. Synaptic dysfunction and oxidative stress in Alzheimer's disease: emerging mechanisms. J Cell Mol Med 2006;10:796-805.

39. Desquiret-Dumas V, Gueguen N, Leman G, et al. Resveratrol induces a mitochondrial complex I-dependent increase in NADH oxidation responsible for sirtuin activation in liver cells. J Biol Chem 2013;288:36662-75.

40. Ungvari Z, Orosz Z, Rivera A, et al. Resveratrol increases vascular oxidative stress resistance. Am J Physiol Heart Circ Physiol 2007;292:H2417-24.

41. Csiszar A, Labinskyy N, Podlutsky A, et al. Vasoprotective effects of resveratrol and SIRT1: attenuation of cigarette smoke-induced oxidative stress and proinflammatory phenotypic alterations. Am J Physiol Heart Circ Physiol 2008;294:H2721-35.

42. Ungvari Z, Labinskyy N, Mukhopadhyay P, et al. Resveratrol attenuates mitochondrial oxidative stress in coronary arterial endothelial cells. Am J Physiol Heart Circ Physiol 2009;297:H1876-81.

43. Milne JC, Lambert PD, Schenk S, et al. Small molecule activators of SIRT1 as therapeutics for the treatment of type 2 diabetes. Nature 2007;450: 712-6.

44. Timmers S, Konings E, Bilet L, et al. Calorie restriction-like effects of 30 days of resveratrol supplementation on energy metabolism and metabolic profile in obese humans. Cell Metab 2011;14:612-22.

45. Lekakis J, Rallidis LS, Andreadou I, et al. Polyphenolic compounds from red grapes acutely improve endothelial function in patients with coronary heart disease. Eur J Cardiovasc Prev Rehabil 2005;12:596-600.

46. Wong RH, Howe PR, Buckley JD, Coates AM, Kunz I, Berry NM. Acute resveratrol supplementation improves flow-mediated dilatation in overweight/obese individuals with mildly elevated blood pressure. Nutr Metab Cardiovasc Dis 2011;21:851-6.

47. Li H, Dai M, Jia W. Paeonol attenuates high-fat-diet-induced atherosclerosis in rabbits by anti-inflammatory activity. Planta Med 2009;75:7-11.

48. Li H, Xie YH, Yang Q, et al. Cardioprotective effect of paeonol and danshensu combination on isoproterenol-induced myocardial injury in rats. PLoS One 2012; 7:e48872. 


\section{Articles | Guietal.}

49. Sipos PI, Rens W, Schlecht $\mathrm{H}$, et al. Uterine vasculature remodeling in human pregnancy involves functional macrochimerism by endothelial colony forming cells of fetal origin. Stem Cells 2013;31:1363-70.

50. Egorova AD, DeRuiter MC, de Boer HC, et al. Endothelial colony-forming cells show a mature transcriptional response to shear stress. In Vitro Cell Dev Biol Anim 2012;48:21-9.

51. Fioretta ES, Simonet M, Smits AI, Baaijens FP, Bouten CV. Differential response of endothelial and endothelial colony forming cells on electrospun scaffolds with distinct microfiber diameters. Biomacromolecules 2014; 15:821-9.
52. Joo HJ, Song S, Seo HR, et al. Human endothelial colony forming cells from adult peripheral blood have enhanced sprouting angiogenic potential through up-regulating VEGFR2 signaling. Int J Cardiol 2015; 197:33-43.

53. Grundmann M, Haidar M, Placzko S, et al. Vitamin D improves the angiogenic properties of endothelial progenitor cells. Am J Physiol Cell Physiol 2012;303:C954-62.

54. Skokowa J, Lan D, Thakur BK, et al. NAMPT is essential for the G-CSFinduced myeloid differentiation via a $\mathrm{NAD}(+)$-sirtuin-1-dependent pathway. Nat Med 2009;15:151-8. 